OnLine Journal of Biological Sciences 8 (1): 15-18, 2008

ISSN 1608-4217

(C) 2008 Science Publications

\title{
Er: YAG Laser Device for Taking Blood Samples
}

\author{
${ }^{1}$ José L. Cabrera, ${ }^{2}$ Luis Ponce, ${ }^{2}$ Miguel Arronte, ${ }^{2}$ Teresa Flores, \\ ${ }^{1}$ Bradies Lambert and ${ }^{2}$ Adrián Peña \\ ${ }^{1}$ Institute of Materials and Reagents, \\ Havana University, Zapata y G, Vedado 10400, C. Havana, Cuba \\ ${ }^{2}$ Research Center for Applied Science and Advanced Technology, \\ Altamira, Km 14,5 Carretera Tampico-Puerto Industrial, Altamira 89600, Mexico
}

\begin{abstract}
Problem statement: Secure methods for blood sampling which is performed at present by metallic lancets, has been a concern in the modern medicine in order to diminish the risk of contamination. A novel solution has appeared recently and consists in the use of laser pulses which ablates a tiny hole in skin. Approach: A laser device with Er: YAG active medium for skin perforation with focal point detection by light wall, is presented in this work. Results: This device permits the capture of blood samples without any mechanical contact with human skin. It includes a pulsed Er:YAG laser emitting at $2.94 \mathrm{~nm}$ and a sensor which determine the focal point of laser light on the skin surface in order to optimize the hole dimensions between 40 and $60 \mu \mathrm{m}$ by using pulses with energy between $300 \mathrm{~mJ}$ and $550 \mathrm{~mJ}$ according the skin thickness. Conclusions: This new device allows to eliminate the use of metallic lancets that cause pain and can provoke the diseases transmission.
\end{abstract}

Key words: Laser, lancet, skin, perforation, blood

\section{INTRODUCTION}

Many investigations are performed in order to develop new equipments and methods for the analysis that eliminate the risks of spread and diminish the pain. This purpose can be applied to blood analysis, one of more extended methods of analysis that permits to diagnose different diseases and can also offer an overview of the organism functions. In order to perform these analyses it is necessary to draw blood from the body, with the corresponding risk of contracting or transmitting any type of diseases.

On a recent date a new solution has appeared and that consists in device that uses laser pulses which ablates a tiny hole through the external layers of the skin $^{[1]}$. The laser shows the important advantage of eliminating mechanical contact, with the subsequent decrease in the risk of contamination. On the other hand the use of short pulses reduces tissue trauma that the conventional metal lancet produces and allows to diminish of pain greatly.

The best candidate for this application is the Er: YAG laser. The wavelength of this laser is $2.94 \mu \mathrm{m}$, with is matching with water's absorption peak. Taking in to account that the water is the major constituent of organic tissue, it's possible to explain the strong absorption of tissue for this wavelength ${ }^{[2]}$.

On the other hand, due this strong absorption in water, this laser exhibits a little penetration depth. In comparison with Ho: YAG or Nd: YAG laser, the ER:YAG have a smaller penetration that is very convenient to obtain a bigger accuracy in perforation.

For ER:YAG laser, the photo-ablation process occur when the tissue is irradiated with high-power density radiation (about $10^{8} \mathrm{~W} \mathrm{~cm}^{-2}$ ) with a short pulses less than $1 \mathrm{~ms}$ duration. With these parameters, it is possible to vaporize the tissue by producing mechanical stress, which decomposes the matter in small particles. This micro-explosion is strongly localized causing minimal stress to the surrounding area ${ }^{[2]}$.

Specifically the thermal character of the interaction for the Er: YAG wavelength offers an additional advantage: Provokes a rapid tissue photocoagulation avoiding the unnecessary blood loss after getting the sample ${ }^{[3,4]}$.

\section{MATERIALS AND METHODS}

The principle for operating the device is very simple: A laser pulse of high intensity performs the

Corresponding Author: José L. Cabrera, CICATA-IPN, Altamira, Km 14, 5 Carretera Tampico-Puerto Industrial, Altamira 89600, México 
ablation of tissue in a specific area, so that opening of a hole through which a blood sample obtaining can be achieved.

The device for skin perforation has the following parts: The laser power supply and the ER: YAG laser head that includes the optical focusing system (Fig. 1). The last one is contained in the hand-held manipulator that permits the easy access to the different parts of human body.

Laser head: In the laser head of our device the pump radiation is produced by xenon pulse lamp. The light from this lamp is concentrated on the active medium (ER: YAG rod) through an special reflector. A monolithic quartz reflector with external metallic coating is used. The quartz block is doped with atomic $\mathrm{Ce}$ at $1 \%$ doping level. This feature avoids the optical damage (solarization) of the active medium due the ultraviolet light emitted by xenon lamp. On the other hand, the conversion of UV to visible radiation contributes to enhance the absorption of pump light in ER: YAG crystal and, in consequence, the efficiency of this laser.

With the purpose of obtaining a compact device and at the same time to decrease the costs for its manufacture, the resonator mirrors of 99.8 and $85 \%$ reflectance respectively, were placed directly above the rod ends. The rod dimensions are $5 \times 50 \mathrm{~mm}$. A $25 \mathrm{~mm}$ diameter $\mathrm{CaF}_{2}$ lens of $35 \mathrm{~mm}$ of focal distance focuses the laser radiation in the point where the perforation is performed.

Power supply: The configuration of the power supply has a typical design for these kinds of lasers. It consists of a capacitor charger, trigger circuit and control unit. The discharge of the capacitor is induced by a trigger circuit. This discharge provokes the emission of light pump pulse. The maximum pulse energy that the lamp emits is $16 \mathrm{~J}$ which produce a laser emission of about $0.5 \mathrm{~J}$. The trigger is similar to the one used in a photographic flash, witch can be compact one at a very low cost.

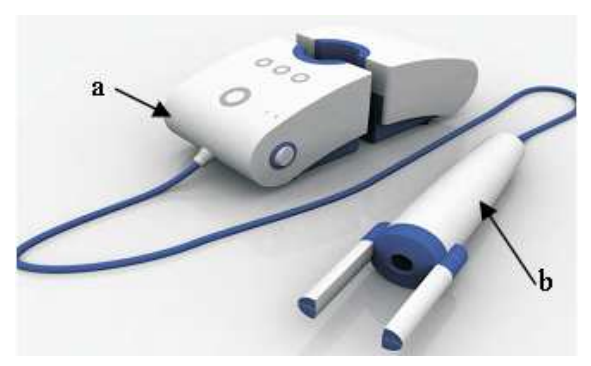

Fig. 1: View of laser skin perforator. (a): Power supply, (b): Laser head
In Fig. 2, we can observe different elements of the laser device. At the left side of the picture, we can see the laser rod, the lens, the mirrors and the reflector cavity. At the right side we can see the power supplies.

Focal point detector by light wall: It was used a light wall focal point detector in order to accurately determine the position of skin surface respect the focal point.

In Fig. 3 it's showed the configuration of this device. Two basic elements integrate the sensor: The light emitter diode that produces the reference red light and the photodiode that receives directly the light from the emitter. The visible red light permits to locate the finger in the correct position. The external border of the light wall is located at $35 \mathrm{~mm}$ from the $\mathrm{CaF}_{2}$ lens in coincidence with focal point. When the skin surface cross the light wall, the device generate the signal to immediately shoot the laser and make the perforation.

The accuracy of this configuration makes possible to obtain a holes with $200 \mu \mathrm{m}$ in diameter or less. This patented solution is very important approaches that guarantee the pain diminish and control the volume of blood to be extracted.

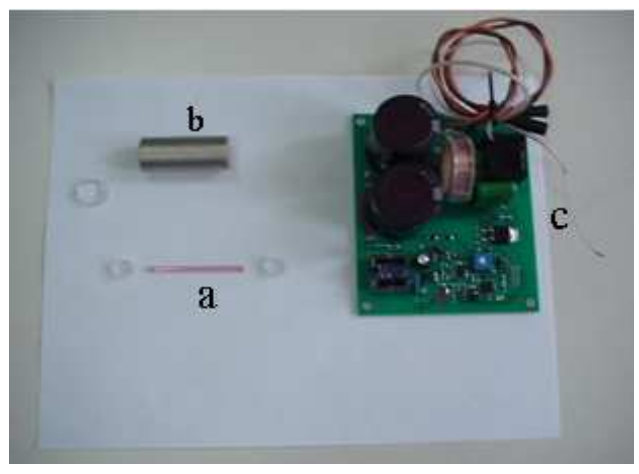

Fig. 2: Photograph of the internal laser parts. (a): Laser rod, (b): Reflecting cavity, (c): Power supply board

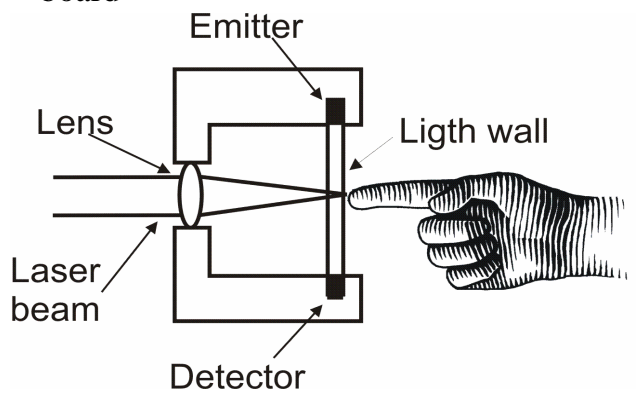

Fig. 3: Schematic of focal point detector 
Taking samples: The device has a compact design integrated by two parts: The power supply unit and the hand-held laser device. On the power supply box, the control panel shows three switches that correspond to three different level of laser pulse energy. This feature is used to compensate the variations in thickness of the human skin. Higher values may be used for patients with thicker skin while lower values may be used for patients with thin or tender skin. The pulse energy can be controlled in range $300-500 \mathrm{~mJ}$. When the charging level (corresponding to energy level selected according skin thickness) is reached, pressing again the switch the laser can be ready to produce one pulse.

To initialize the device the push switch must be pressed in order to start the capacitor charger. After this operation, the switch of selected energy level could be pressed. By using the hand-held device, the physician aims the red reference light to the skin surface. The patient should move the skin surface to the focal point position that is detected when the light well is interrupted. Only when the surface is located in the correct position on the focal point, the signal from detector send the shot order and the laser pulse is emitted. This procedure guarantees the process without any contact and avoids the risks of accident by casual incidents.

The device produces a perforation with a typical diameter between 100 and $200 \mu \mathrm{m}$ in less than $0.3 \mathrm{~ms}$. The obtained perforation leaves the most external blood vessels exposed to allow for the blood taken out.

Safety aspects: Laser ablation process of skin is known to produce airborne contaminants including carbon particulates and gaseous hydrocarbons. Previous works about laser ablation byproducts, based on surgical laser applications, have no resulted in the detection of contaminants above recommended limits. It should be consider that these cases are related to the ablation of tissue volumes many hundreds of time greater than the volume of ablation produced by our device. The literature related with ER: YAG laser perforator, demonstrate that no viable virus, papillomavirus or bacteria are detected in the composition of ejected material during the tissue ablation ${ }^{[5]}$.

\section{RESULTS}

The measurement of laser energy and the pulse duration was performed. The pulse energy, for the maximum value of pumping was about $550 \mathrm{~mJ}$. This measurement was performed using the energy meter
LM-09 with pyroelectric detector. Pulse duration was measured with a Thorlabs Inc. model 201/579-7227 photodiode and Textronix TS540A oscilloscope. The device can be operated with a maximum frequency on 2 pulses per minute. In Fig. 4, it is showed the form of laser pulsed obtained in oscilloscope. The average pulse duration was $180 \mu \mathrm{s}$.

The output energy versus pump energy dependence is showed in Fig. 5. The three selected point references the three real levels of pulse energy used to different skin thickness. As the figure show, these levels are located on the linear zone. For our configuration, a 3\% slope efficiency is obtained, that is consider an excellent result for Er:YAG laser ${ }^{[6]}$.

In Fig. 6, a photograph of perforation is showed. The diameter of this perforation is about $0.2 \mathrm{~mm}$, the crater looks clean without debris or cuts.

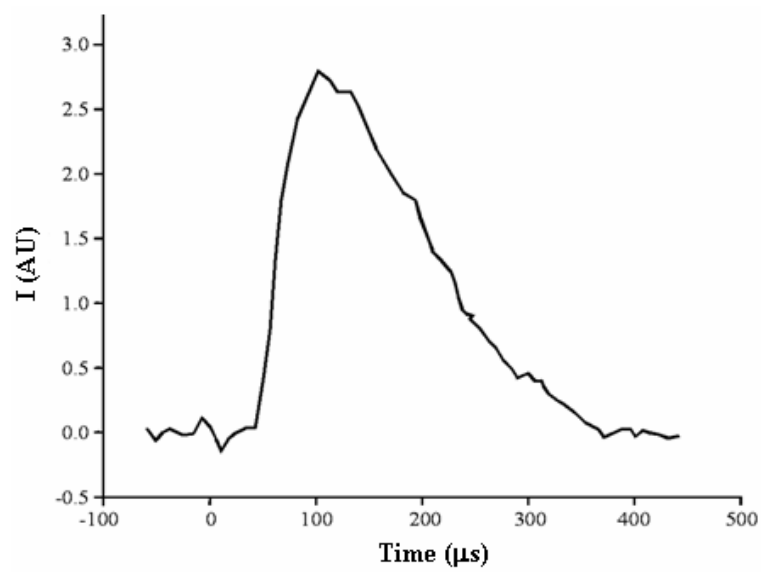

Fig. 4: The measurement of laser pulse duration

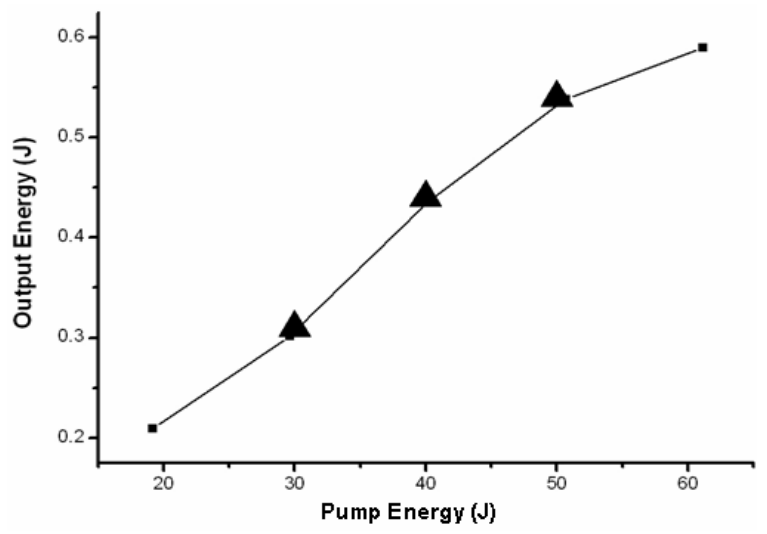

Fig. 5: Experimental results for output energy versus pump energy 


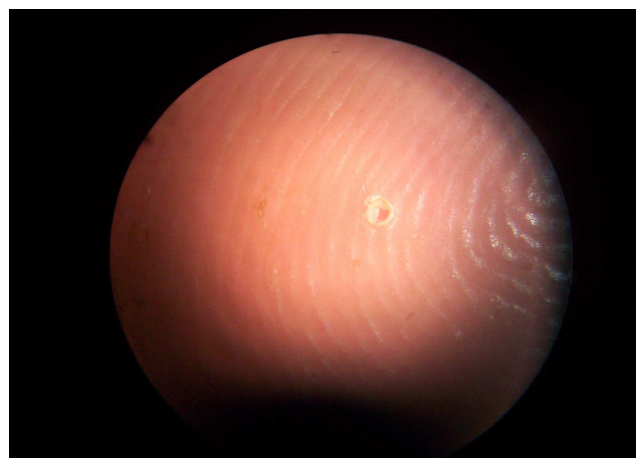

Fig. 6: View at microscope of perforation of finger made by Er: YAG laser. Diameter of perforation $0.2 \mathrm{~mm}$

\section{DISCUSSION}

The results of our investigation demonstrates that it is possible to realize the skin perforation to obtain blood samples by means of laser pulses, achieving perforations with the adequate diameter.

One important aspect to be consider for the efficient use in the clinical practice, is the differences in skin thicknesses. Though the equipment has three levels of pulse energy for irradiating thin, average and thickly skins, the practice demonstrates that it is difficult to determine the needed energy only from the visual observation

To solve this problem, the authors are working in the development of an optical system for the determination, at least in qualitative form, of skin thickness in the region where the perforation will be performed.

This solution would allow to achieve, in principle, that the equipment could programme automatically, the energy needed for any skin thickness. By this way, the use of laser energy will be optimized and diminished the pain caused by an excessive dose of energy.

\section{CONCLUSION}

The laser skin perforator has several advantages above traditional lancets: Significant decrease in the risk of contamination, diminish of pain and the elimination of the needless. The design with wall light focal point detector guarantees the accuracy in perforation and eliminates any contact and therefore avoids the risk of contamination.

In resume, we have developed a laser skin perforator to be used in order to create a small and clean hole that penetrates in to the capillary bed, permitting to obtain a blood sample for analytic purposes. The perforations can be obtained in human skin for capture of blood samples, by using a pulse energy selection in correspondence with the skin thickness. The device is compact, ergonomic and can be use in clinic or at home.

\section{AKNOWLEDGMENT}

This study was supported by CONACYT.

\section{REFERENCES}

1. Ponce, L., M. Arronte, J.L. Cabrera and T. Flores, 2006. Laser skin perforator with focal point detection. Proceeding of the SPIE International 5th Symposium on Optics Industry, Feb. 2006, The International Society for Optical Engineering, USA., pp: 604604-604608. DOI: 10.1117/12.674379.

2. Majaron, B., P. Plestenjak and M. Lukac, 1999. Thermo-mechanical laser ablation of soft biological tissue: Modeling the micro-explosions. Applied Phys. B-Lasers Optics, 69: 71-80. DOI: 10.1007/s003400050772.

3. Walsh, T., T.J. Flottea and T.F. Deutsch, 1989. Er:YAG laser ablation of tissue: Effect of pulse duration and tissue type on thermal damage. Lasers Surg. $\quad$ Med., $\quad 9$ : 314-326. http://www.medscape.com/medline/abstract/27613 27?src=emed_ckb_ref_0.

4. Jacques, S.L. and S.A. Prahl, 1987. Modeling optical and thermal distributions in tissue during laser irradiation. Lasers Surg. Med., 6: 494-503. http://www.bme.ogi.edu/ prahl/pubs/abs/jacques87 a.html.

5. Hughes, P.S. and A.P. Hughes, 1998. Absence of human papillomavirus DNA in the plume of erbium:YAG laser-treated warts. J. Am. Acad. Dermatol., $\quad 38$ : $\quad$ 426-428. http://www.ncbi.nlm.nih.gov/pubmed/9520024.

6. Brunken, A., L. Casper, H. Genz, C. Hessler and S. Khodyachykh, 2003. Influence of doping the pump-chamber material in a flashlamp pumped Er: YAG laser. Optics Laser Technol., 35: 331-333. DOI: 10.1016/S0030-3992(03)00005-7. 